\title{
Tuning Structural and Mechanical Properties of Two-Dimensional Molecular Crystals: The Roles of Carbon Side Chains
}

\author{
Huanyao Cun, ${ }^{\dagger}$, Il Yeliang Wang, ${ }^{*}, \dagger, \mathbb{I}$ Shixuan Du, ${ }^{, \dagger,}, \mathbb{I}$ Lei Zhang, ${ }^{\dagger}$ Lizhi Zhang, ${ }^{\dagger}$ Bing Yang, ${ }^{\dagger}$ Xiaobo He, ${ }^{\dagger}$
} Yue Wang, ${ }^{\ddagger}$ Xueyan Zhu, ${ }^{\S}$ Quanzi Yuan, ${ }^{\S}$ Ya-Pu Zhao, ${ }^{\S}$ Min Ouyang," Werner A. Hofer, ${ }^{\perp}$ Stephen J. Pennycook, ${ }^{\#}$ and Hong-jun Gao ${ }^{\dagger}$

${ }^{\dagger}$ Institute of Physics, Chinese Academy of Sciences, Beijing 100190, China

${ }^{\ddagger}$ Key Lab of Supramolecular Structure and Materials, Jilin University, Changchun 130023, China

${ }^{\S}$ Institute of Mechanics, Chinese Academy of Sciences, Beijing 100190, China

"Department of Physics, University of Maryland, Maryland 20742, United States

${ }^{\perp}$ Stephenson Institute for Renewable Energy, The University of Liverpool, Liverpool, L69 3BX, Umited Kingdom

${ }^{\#}$ Materials Science and Technology Division, Oak Ridge National Laboratory, Oak Ridge, Tennessee 37831, United States

\section{Supporting Information}

ABSTRACT: A key requirement for the future applicability of molecular electronics devices is a resilience of their properties to mechanical deformation. At present, however, there is no fundamental understanding of the origins of mechanical properties of molecular films. Here we use quinacridone, which possesses flexible carbon side chains, as a model molecular system to address this issue. Eight molecular configurations with different molecular coverage are identified by scanning tunneling microscopy. Theoretical calculations reveal quantitatively the roles of different molecule-molecule and molecule-substrate interactions and predict the observed

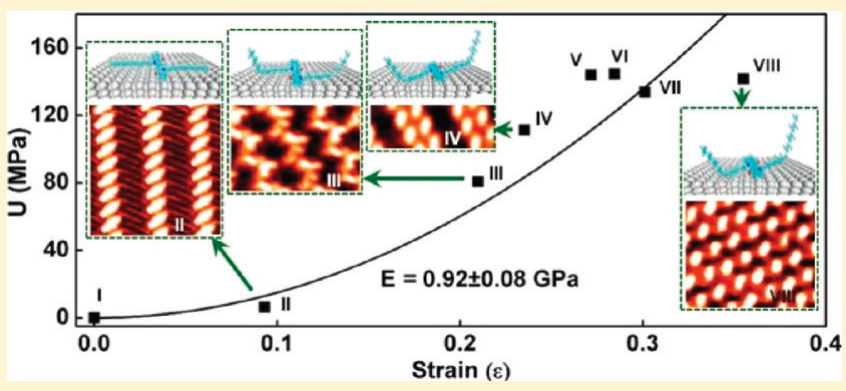
sequence of configurations. Remarkably, we find that a single Young's modulus applies for all configurations, the magnitude of which is controlled by side chain length, suggesting a versatile avenue for tuning not only the physical and chemical properties of molecular films but also their elastic properties.

KEYWORDS: Two-dimensional molecular crystal, molecular side chain, elastic properties, self-assembly, scanning tunneling microscopy

Organic-molecule-based flexible electronics has been of significant interest due to its potential to challenge conventional silicon-based technologies. ${ }^{1-4}$ Crucial to their applicability is resilience of the physical properties of organic compounds and films to mechanical deformation, which requires an in-depth understanding and control of film structure and elastic properties at the molecular level. At present, however, there is little understanding of the underlying mechanisms determining the mechanical properties and the ensuing dimensionality of organic films ${ }^{5-7}$ despite the fact that molecular self-assembly has been extensively investigated on solid surfaces. ${ }^{8,9}$ In this work, $N, N^{\prime}$-dihexadecyl-quinacridone $\left(\mathrm{C}_{52} \mathrm{H}_{76} \mathrm{O}_{2} \mathrm{~N}_{2}\right.$ : QA16C, Figure 1A) was chosen as a model molecule because quinacridone (QA) and its derivatives are well-known as stable organic pigments and dopant emitters, possessing high chemical stability with technologically important photovoltaic and photoconductive properties. ${ }^{10-13}$ In particular, the QA16C molecule has two long carbon side chains, allowing for a subtle interplay of the various moleculemolecule and molecule-substrate interactions that may lead to different possible molecular configurations on a surface. The side chain may also affect the dimensionality of the system, providing one-dimensional or two-dimensional molecular assembly patterns. Scanning tunneling microscopy (STM) is used to identify molecular configurations and to determine their evolution with systematic variation of molecular coverage at single molecular scale. We observe a remarkable sequence of stable and distinct configurations, which agree well with theoretical prediction. Annealing experiments are used to separate energetically stable configurations from metastable configurations. Combining the experimental data with firstprinciples and force-field calculations we are able to quantitatively determine interaction energies and to extract elastic properties of the molecular monolayer.

Methods. Experimental Section. All experiments were carried out in an ultrahigh vacuum (UHV) system equipped with molecular evaporators and a variable temperature scanning tunneling microscope (Omicron, Germany) with the base

Received: October 12, 2011

Revised: February 22, 2012

Published: February 29, 2012 


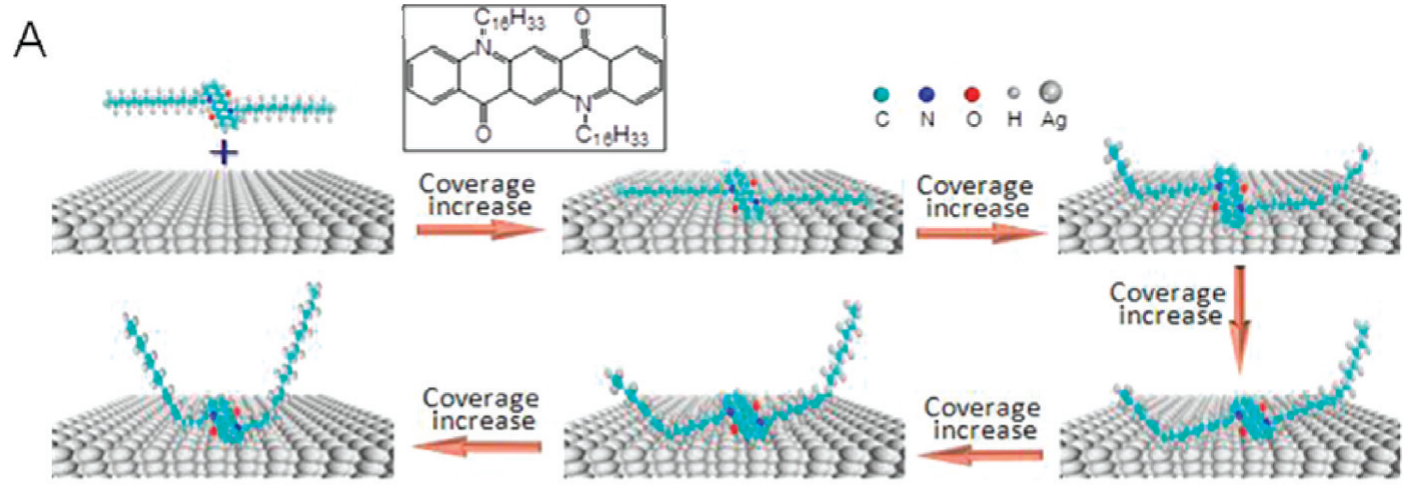

B

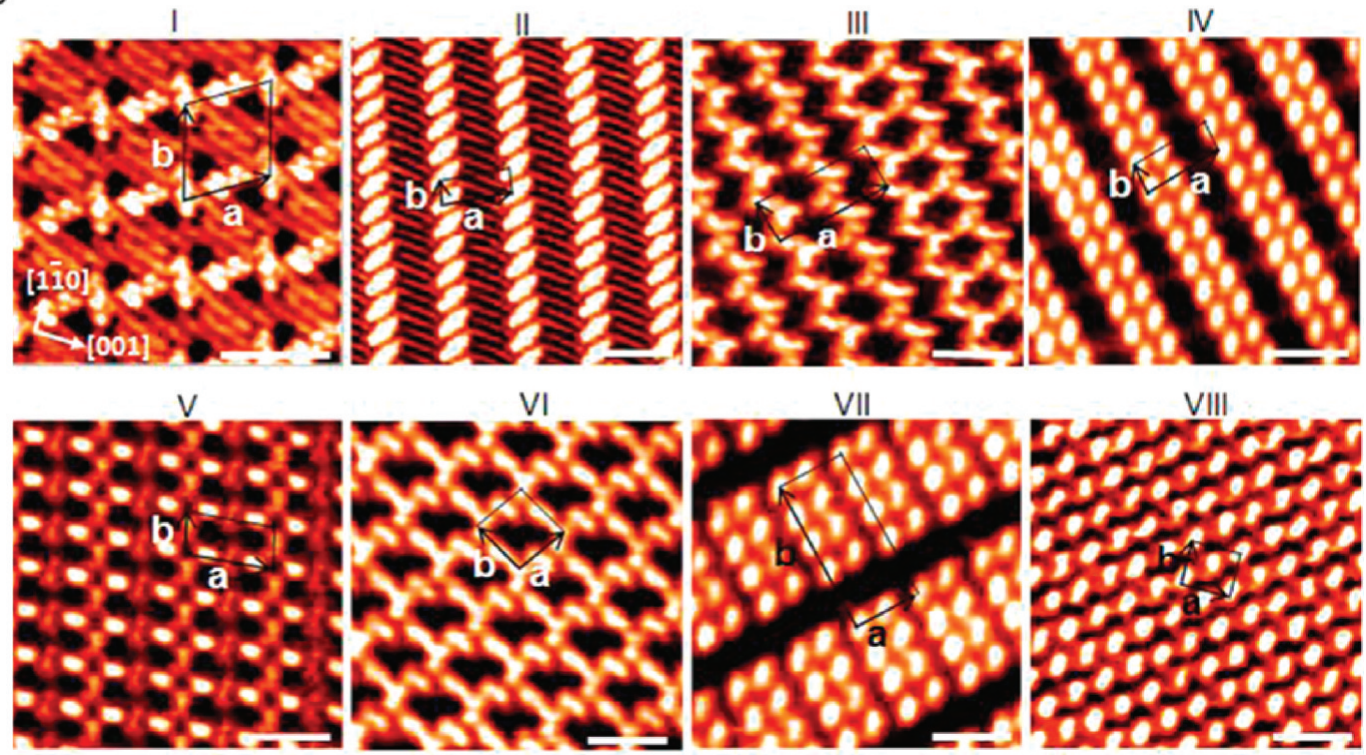

Figure 1. Evolution of QA16C molecular self-assembly structures on the $\mathrm{Ag}(110)$ surface with increasing coverage. (A) Schematics of the change of the molecular configuration with increasing QA16C coverage. Inset shows the chemical structure of QA16C. (B) High-resolution STM images of eight different structures of QA16C molecules on $\mathrm{Ag}(110)$. Structures I and II are obtained at low molecular coverage with the alkyl chains of the molecules clearly resolved. For structures III-VIII, only the molecular backbones are visible. The unit cells are indicated with solid lines. The scanning parameters of each image are I, $-1.03 \mathrm{~V}, 0.40 \mathrm{nA}$; II, $-1.00 \mathrm{~V}, 0.11 \mathrm{nA}$; III, $-1.03 \mathrm{~V}, 0.10 \mathrm{nA}$; IV, $-1.05 \mathrm{~V}, 0.08 \mathrm{nA}$; $,-1.00 \mathrm{~V}, 0.08 \mathrm{nA}$; VI, $-1.03 \mathrm{~V}, 0.08 \mathrm{nA}$; VII, $-1.05 \mathrm{~V}, 0.08 \mathrm{nA}$; VIII, $-1.03 \mathrm{~V}, 0.08 \mathrm{nA}$. The scale bar is $3 \mathrm{~nm}$.

pressure of $2.0 \times 10^{-10}$ mbar. The single crystal $\mathrm{Ag}(110)$ (orientation accuracy $<0.1^{\circ}$, MaTeck Company) has been prepared in vacuum by repeated cycles of $\mathrm{Ar}^{+}$sputtering and subsequent annealing, as described previously. ${ }^{14}$ QA16C molecules in powder form were purified by a sublimation process at the range of $10^{-7} \mathrm{mbar}^{15}$ followed by degassing at $440 \mathrm{~K}$ for several hours under UHV conditions, which removed more volatile impurities sufficiently. The QA16C molecules were evaporated with different evaporation times from organic molecular beam epitaxial cell while keeping the $\operatorname{Ag}(110)$ substrate at room temperature. The temperature of the cell was held at $460 \mathrm{~K}$ as measured with a thermocouple contacted with the cell. The as-prepared sample was transferred from the sample-preparation chamber to the STM chamber. Then STM measurements were carried out with an electrochemically etched tungsten tip. Several times, the sample was returned to the evaporation stage where more molecules were dosed onto the surface, and after each step of deposition further STM measurements were carried out. All STM images were taken in constant-current mode at room temperature.
Computational. In order to determine the interactions between molecular backbones and substrate, the density functional theory (DFT) calculations used the PerdewBurke-Ernzerhof (PBE) flavor of the generalized gradient approximation $(\mathrm{GGA})^{16}$ in an all-electron plane-wave formulation (projector augmented wave method) as implemented in the VASP code. ${ }^{17,18}$ The molecule- $\operatorname{Ag}(110)$ system is modeled within the supercell approach and contains four atomic layers of silver with adsorbed molecules on one side of the slab. It is generated with the theoretical bulk silver lattice parameter of $4.116 \AA$ and a $p(5 \times 7)$ in-plane surface unit cell. During our ab initio calculations, the uppermost two silver layers and the molecular atoms are allowed to relax until the atomic forces are lower than $0.02 \mathrm{eV} / \AA$. We apply the Langreth-Lundqvist functional van der Waals (vdW) calculations $^{19}$ and obtain a value $(\sim 450 \mathrm{meV})$, which perfectly accounts for the co-occurrence of different ordering patterns of the molecules (III and VIII, see further down). The adsorption energy of either carbon chain or backbone on the substrate $\left(E_{\mathrm{ad}}\right)$ is calculated using the total energy of the adsorbate on the 
Table 1. Structural Parameters of the Eight QA16C Structures Observed on $\operatorname{Ag}(110)$

\begin{tabular}{|c|c|c|c|c|c|c|c|c|}
\hline Structure & I & II & III & IV & $\mathrm{V}$ & VI & VII & VIII \\
\hline Matrix notation & $\left(\begin{array}{cc}5 & 5 \\
-2 & 9\end{array}\right)$ & $\left(\begin{array}{cc}6 & 5 \\
-1 & 3\end{array}\right)$ & $\left(\begin{array}{cc}9 & 10 \\
-3 & 5\end{array}\right)$ & $\left(\begin{array}{cc}6 & 9 \\
-2 & 3\end{array}\right)$ & $\left(\begin{array}{rr}11 & 2 \\
-1 & 6\end{array}\right)$ & $\left(\begin{array}{cc}3 & 7 \\
-5 & 5\end{array}\right)$ & $\left(\begin{array}{cc}5 & 6 \\
-10 & 14\end{array}\right)$ & $\left(\begin{array}{ll}5 & 0 \\
0 & 6\end{array}\right)$ \\
\hline $\begin{array}{c}\text { Number of } \\
\text { molecules per unit } \\
\text { cell }\end{array}$ & 2 & 1 & 4 & 2 & 4 & 3 & 8 & 2 \\
\hline $\begin{array}{l}\text { Area per unit cell } \\
\qquad\left(\mathrm{nm}^{2}\right)\end{array}$ & 6.49 & 2.72 & 8.86 & 4.25 & 8.03 & 5.9 & 15.35 & 3.54 \\
\hline $\begin{array}{c}\text { Number of } C \\
\text { atoms adsorbed on } \\
\mathrm{Ag}(110) \text { per side } \\
\text { chain }\end{array}$ & 16 & 16 & 11 & 10 & 8 & 8 & 8 & 6 \\
\hline $\begin{array}{c}\text { Schematic of } \\
\text { molecules in one } \\
\text { unit cell }\end{array}$ & & & & & & & & 0 \\
\hline
\end{tabular}

substrate $\left(E_{\mathrm{t}}\right)$ minus the total energies of the adsorbate $\left(E_{\mathrm{a}}\right)$ and the substrate $\left(E_{\mathrm{s}}\right), E_{\mathrm{ad}}=E_{\mathrm{t}}-\left(E_{\mathrm{a}}+E_{\mathrm{s}}\right)$.

The $\mathrm{MM}+$ force field is employed to investigate $\mathrm{vdW}$ interactions between carbon chains (CC) and substrate, and also between neighboring CC. As for the interactions between $\mathrm{CC}$ and substrate, the model is built with a $\mathrm{p}(7 \times 12)$ in-plane surface unit cell and the substrate contains four atomic layers of silver with the adsorbing carbon chain on one side of the slab. Since the lengths of adsorbing CC in the eight structures are different, the number of carbon atoms in the chain decreases from 16 to 8 . For the interactions between neighboring CC, we built a model in which two $\mathrm{CC}$ are aligned parallel in vacuum, and the number of carbon atoms varies from 1 to 8 . For the calculation of vdW interactions between CC and substrate, only the CC are allowed to relax until the system energy gradient was lower than $0.1 \mathrm{kcal} / \mathrm{mol}$.

Results and Discussion. STM images of the molecular assembly at different coverage are shown in Figure 1B (large scale images are shown in Figure $S 1$ in Supporting Information). Eight different structures in total can be unambiguously identified and their evolution is summarized as follows. Initial deposition on a clean $\mathrm{Ag}(110)$ surface yields two coexisting structures of a network (I) and a line structure (II). The aromatic backbones as well as the alkyl chains are clearly distinguished as bright protrusions and dim stripes, respectively. As additional molecules are deposited, a herringbone-like pattern (III) emerges, while the surface areas of I and II are gradually diminished. Subsequently, patterns of dimer rows (IV) and braids (V) form and coexist with pattern III. Upon the addition of more molecules, domains of pattern $\mathrm{V}$ become bigger while a new meshlike structure (VI) also starts manifesting itself. Further deposition induces the formation of a tetramer structure (VII). Finally, the molecular adlayer develops into a close-packed structure (VIII), which covers almost the entire surface (Supporting Information Figure S1). In all these high-resolution images each bright elliptic protrusion can be routinely assigned to the backbone of one QA16C molecule based on size and shape. ${ }^{20}$ The structural parameters of all eight different patterns can be determined from Figure $1 \mathrm{~B}$ and are summarized in Table 1 and Supporting Information Table S1.

In the patterns of I and II in Figure 1B, both backbones and alkyl chains can be resolved, and the alkyl chains are mutually parallel and uniformly oriented on the substrate. Similar motifs have been previously reported from the self-assembly of long alkyl chain molecules at the liquid/graphite interface, ${ }^{21-25}$ as well as for QA16C molecules on the metal substrates $\mathrm{Cu}(110)^{20}$ and $\mathrm{Au}(111) .{ }^{26}$ However, the angles between the backbones and the alkyl chains differ between I and II: 54 and $132^{\circ}$ for I, $108^{\circ}$ for II. These data highlight the flexibility of alkyl chains, that is, a clear response to the change of local substrate coverage by self-reorientation.

As compared with patterns I and II, patterns III-VIII only manifest molecular backbones in the high-density STM motifs with an absence of molecular side chains. One possibility is that the side chains could be separated from the molecular backbones and lost (completely or partially) during the thermal evaporation. However, considering the fact that deposition temperature remains the same for all configurations (from I to VIII) in Figure 1B, this possibility can be safely excluded. By an in-depth analysis, we found that the spacing between neighboring molecules in III-VIII becomes smaller than those in I and II, which qualitatively suggests that a part of the molecular side chains may be tilted away from the substrate under the condition of high molecular coverage, as schematically illustrated for structure changes in Figure 1A. The tendency to form higher density structures at higher molecular coverages has been previously reported, for example, a similar observation with molecules containing alkyl chains at the liquid-solid interface, where the multiple structures depend on the molecular concentrations. ${ }^{27}$

In order to quantify and understand the evolution of molecular structures with different substrate coverage, we calculate molecular packing densities of the eight structures based on the STM images. The calculated results are shown in Supporting Information Table S1 and plotted in Supporting Information Figure $\mathrm{S} 2$. We find that the eight different configurations can be classified into two different groups, that is, a low-density group (I and II) and a high-density group (III-VIII) with an obvious difference of packing density; the difference is $23 \%$ between II and III, while it is on average $7.6 \%$ between any other adjacent configurations.

Except for their packing density, there exist two other distinct differences between these two groups: (i) in the low density group, both backbone and alkyl chains can be well resolved in the corresponding STM images, while only the backbones are visible in the structures in the high density group; (ii) the average area occupied by one molecule is either larger (low density group) or smaller (high density group) than the area of the molecule, which is $2.99 \mathrm{~nm}^{2}$ including the extended alkyl chains. For example, in III the area is only $2.22 \mathrm{~nm}^{2}$. This feature of III is also confirmed by the short distance between 
two adjacent molecular rows, which is about $1.30 \mathrm{~nm}$, that is, $31.6 \%$ shorter than the length of a free alkyl chain $(1.90 \mathrm{~nm})$. Therefore we conclude that the alkyl chains do not lie flat on the substrate for the six high-density structures (III-VIII, Figure 1B) but are partially uplifted from the surface and reach into the vacuum. However, the fact that side chain must remain partially on the surface due to a steric effect, which can be supported by comparing packing densities of QA16C and shorter chain molecules (QA1C and QA4C, the models and STM images are shown in Supporting Information Figure S3). We have determined the total number of carbon atoms in the side chain that remain on the substrate for six structures in the high-density group (see the data in Table 1, for example, at least six carbon atoms adsorb on the surface in VIII).

We have shown that different structures have different arrangements of carbon chains. As an example, structure I has all carbon chains lying flat on the substrate and structure VIII has the carbon chains partially uplifted, thus representing two different elastic states of the molecular films. Our present study therefore provides a unique way to quantitatively evaluate the elastic properties of these molecular adlayers. The stress-strain relationship of an organic thin film can be approximated as a $2 \mathrm{D}$ isotropic system with a strain energy density $U=[E(1-v$ $\left.\left.-1 / 2 v^{2}\right) /\left(1-2 v^{2}\right)\right] \varepsilon^{2}$, where $E$ is the Young's modulus, $v$ is the Poisson's ratio, and $\varepsilon$ is the strain. ${ }^{28}$ The Poisson's ratio we used for our calculation is $0.3,^{28,29}$ thus this equation can be further simplified to $U=1.6375 E \varepsilon^{2}$. The strain $\varepsilon$ can be determined by $\varepsilon(i, j)=\left(S_{i}{ }^{1 / 2}-S_{j}^{1 / 2}\right) / S_{i}{ }^{1 / 2}$, where $i$ and $j$ denote the different configurations among eight observed structures, $S_{i}$ and $S_{j}$ describe the areas of one molecule in structure $i$ and structure $j$ (Supporting Information Table S1). The strain energy density $U$ can be evaluated as $U(i, j)=\left(E_{a i}-E_{a j}\right) /\left(h_{i} S_{i}\right)$, where $h_{i}$ is the film thickness (see Supporting Information S7 for details of the calculations), and $E_{\mathrm{a} i}$ and $E_{\mathrm{aj}}$ are the adsorption energy in structure $i$ and structure $j$, respectively.

In order to compute the adsorption energies of different structures for an accurate evaluation the mechanical behavior of the films, we calculate all interactions separately and structureby-structure, as summarized in Figure 2. The existence of different percentages of the side chains adsorbed on the substrate for different packing densities strongly suggests that intrinsic interchain and chain-substrate interactions should vary in our observed eight different assembly patterns. Figure $2 \mathrm{~A}$ illustrates the different interactions that should be considered to account for the molecular arrangement. (i) Chemical interaction between molecular backbone and substrate; in our case it is the chemical bonding between oxygen and silver atoms (BB-Ag-chem); (ii) vdW interactions between backbone and substrate $(\mathrm{BB}-\mathrm{Ag})$; (iii) vdW interactions between flat side chains and substrate ( $\mathrm{fC}-\mathrm{Ag}$ ); (iv) $\mathrm{vdW}$ interactions between neighboring uplifted carbon chains (upC-upC); and (v) interactions between neighboring molecular backbones; hydrogen bonds among neighboring backbones have been reported before to result from carboxyl oxygen atoms of one molecule interacting with hydrogen atoms of an adjacent molecule. ${ }^{30,31}$ Considering the distance between oxygen and hydrogen atoms in our adlayers this effect can be safely ignored; thus contribution of $(v)$ is negligible in our evaluation of the total energy.

We now evaluate the above interactions one by one. We find the adsorption energy of a molecule chain with $n$ carbon atoms absorbed on the surface can be described by the relationship $E_{\mathrm{ad}}$ $=137.6-38 n[\mathrm{meV}]$; the corresponding values are summarized
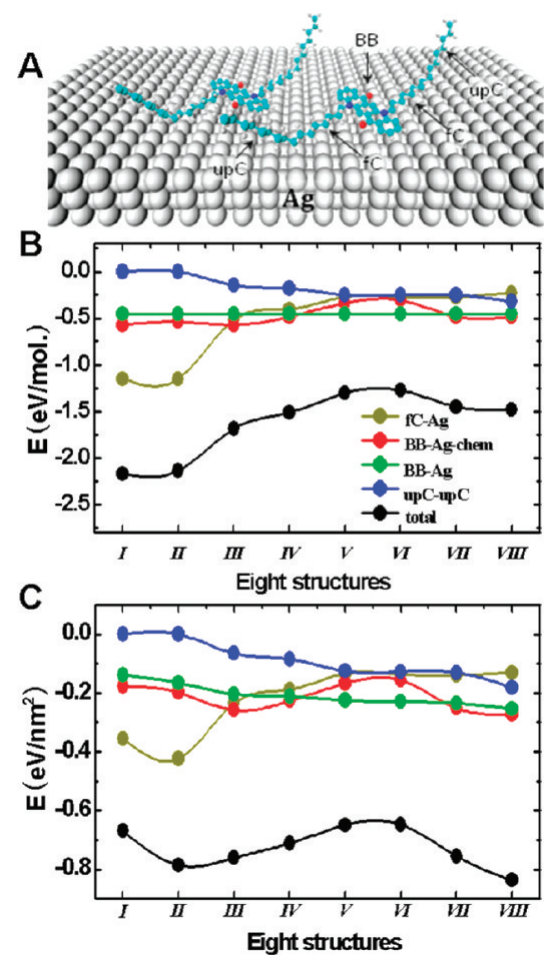

Figure 2. Molecular interactions and total energy calculations. (A) Schematics of the different segments of $\mathrm{QA}$ molecules used to calculate interaction energies. BB represents the QA16C backbone; fC and upC represent flat and uplifted sections of the side chains on the $\mathrm{Ag}(110)$ surface. Interactions include the chemical interaction (BB$\mathrm{Ag}$-chem) and van der Waals interaction (BB-Ag) between backbone and substrate, the interaction between the flat absorbed side-chain and the substrate ( $\mathrm{fC}-\mathrm{Ag})$, and neighboring uplifted chains (upC-upC). (B) Total energy per molecule as a function of the superstructure. (C) Total energy per unit area as a function of the superstructure.

in Supporting Information Table S2. We consider interactions of the molecular backbone with the substrate by optimizing the configuration of a single molecular backbone on $\mathrm{Ag}(110)$. By comparing with experimental STM results (Figure 1B), we observe that there exist two kinds of molecular orientations, corresponding to four molecular configurations by taking into account also the chirality of QA16C molecules on the surface. $^{20,26}$ The optimized models of these four configurations are shown in Supporting Information Figure S4A-D, and the adsorption energy of them is calculated by DFT and given in Supporting Information Table S3. We find that the difference of the energy values among the four adsorption configurations is around $100 \mathrm{meV}$. The increase of the adsorption energy with each carbon atom of the side chain adsorbed on the surface is also about $100 \mathrm{meV}$ (Supporting Information Table S2). This "degeneracy" in energy scales is thus the origin of the observed diversity of structures of QA16C. While the system loses adsorption energy when the carbon chains are uplifted, it also simultaneously gains energy because more molecular backbones can adsorb on the surface. Since the adsorption energies are comparable, the total energy landscape is very flat. Therefore, there exist a large number of possibilities for energy trade-off in structural transformation processes with increasing substrate molecular coverage. The alkyl chains play a key role during the whole structural change process, especially in stabilizing the energetically metastable structures. They not only act as spacers 
$A_{1}$
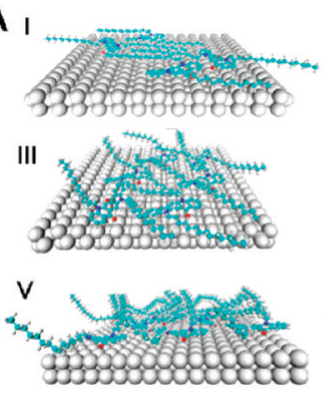

VII

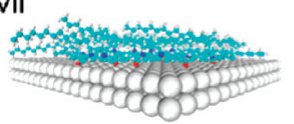

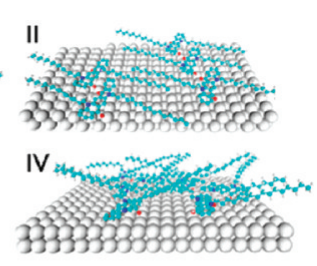

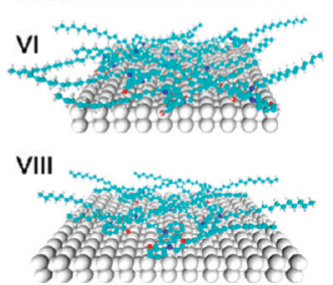

B

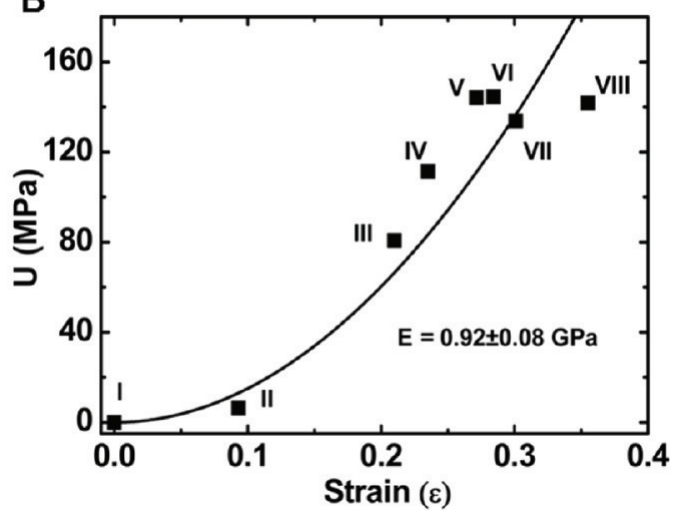

Figure 3. Calculation of the molecular configurations and Young's modulus. (A) Eight molecular configurations on $\mathrm{Ag}(110)$ surfaces. The adsorption sites of the backbone on the surfaces are determined by experimental observations and DFT calculations. The configurations of the side chains are optimized by MM+. (B) Plot of strain energy density $(U)$ vs strain $(\varepsilon)$. The data points from II to VIII stand for the calculated values of $U$ and $\varepsilon$ of the corresponding structure, and each point is calculated from the structure I and itself. The line is a fit to structures I through VIII resulting in an estimated Young's modulus of $0.92 \pm 0.08 \mathrm{GPa}$. The error of the Young's modulus comes from the standard error when we use a quadratic function to fit the $U-\varepsilon$ data.

between two neighboring molecules, ${ }^{32,33}$ but also stabilize a particular adlayer structure due to their interaction ( $\mathrm{fC}-\mathrm{Ag}$ ) with the surface and their mutual vdW interactions (upCupC).

Next, we evaluate the total interaction energy of each observed structure (Figure 2) after considering all the interactions described above. For a molecular crystal, there are generally two different evaluation ways: (i) the total interaction energy per molecule (Figure 2B), and (ii) the total interaction energy per unit area (Figure 2C). A comparison between these two measures has revealed dramatic difference in the predicted energy as well as the stability of different structures. For example, in the low density regime (structures I and II) the energy per molecule is practically identical, while the energy per unit area suggests an energy decrease for structure II. Such differences are also manifest in the high coverage regime. While both the energy per molecule and per unit area suggests a higher stability of structure III with respect to structures IV and V, a preference for structure VIII is predicted by the energy per unit area. Our calculations also demonstrate that the total adsorption energy strongly depends on the weak (upC-upC) interaction, which further highlights the importance of considering all molecular interactions in order to accurately evaluate the mechanical behavior.

In order to examine our theoretical evaluation of the total absorption energy we carried out an annealing experiment to evaluate the stability of the eight structures. The STM observations of the annealed samples indicate that II, III, and VIII are the three most stable and energetically favorable molecular configurations (Supporting Information Figure S5), which agrees very well with the prediction from the energy per unit area calculation. We conclude, therefore, that the energy per unit area is the appropriate measure of energy and can be regarded as the molecular analogue of a surface energy. ${ }^{34,35}$

By combining STM experiments with theoretical calculations, we have obtained the total adsorption energies of different structures and shown that the interaction energy per unit area can be regarded as a surface energy. We also could determine the molecular configurations of the eight observed structures I-VIII by DFT and MM+ simulations, leading to the models shown in Figure 3A. Different configurations have a different thickness $(h)$ caused by the standing-up of the carbon side chains to varying extent. Eight different molecular configurations can be regarded as eight different strain states of the molecular film, and therefore, the strain energy density $(U)$ and strain $(\varepsilon)$ for every structural configuration (I-VIII) can be independently evaluated from our STM measurements and theoretical calculations. By using the formula described above and with the data for $S$ (area of one molecule in observed structure), $E_{\mathrm{a}}$ (total adsorption energy), and $h$ (film thickness), we can easily obtain the values of $U$ and $\varepsilon$ of each individual configuration. Figure $3 \mathrm{~B}$ shows these values and their evolution. We find that the ratio of $U$ to $\varepsilon$ can be fitted by a single formula, including the Young's modulus E.

Remarkably, our data indicate that a single Young's modulus applies to all configurations and the value is $0.92 \pm 0.08 \mathrm{GPa}$. This value is substantially smaller than those of typical conjugated organic-molecule based crystals (e.g., naphthalene and anthracene have Young's modulus of 8.1 and $8.4 \mathrm{GPa}$, respectively). ${ }^{28}$ This is consistent with the fact that the molecules are riding up over one another as they change from one configuration to the next so that the deformation is not strictly elastic and our result represents an effective elastic constant. We also note that dibenzyl ethane has a Young's modulus of $6.3 \mathrm{GPa}^{28}$ which is smaller than naphthalene, due to the ethyl chain. The ethyl chain is only two $\mathrm{CH}_{2}$ groups long, whereas in the QA16C molecule the two carbon chains are sixteen $\mathrm{CH}_{2}$ groups long. These data suggest that the alkyl chains change the Young's modulus considerably. To further confirm the effect of side-chain to the elastic properties, we calculate the Young's modulus of films formed by QA1C and QA4C molecules (this is done theoretically, see Supporting Information 7), that have much shorter side chains compared to QA16C. In this case, the change of total energy of the system is essentially due to the change of intermolecular interactions and does not involve, as in QA16C, a change of conformation of the carbon chains. The Young's modulus for the films of QA1C and QA4C is then $0.15 \pm 0.01 \mathrm{GPa}$ and $0.20 \pm 0.01$ $\mathrm{GPa}$, respectively. This result suggests that the main contribution to the elastic properties of the QA16C monolayer is actually due to conformation changes of the chains. It further demonstrates that the side chain has an important effect on the elastic property of molecular films. As the conformation changes in a molecular layer with varying packing density 
depend on the length of the carbon side chains, we may conclude that this length is a critical factor controlling the Young's modulus of such molecular films. Our results could be extended to other organic compounds with long carbon chains, for example, phthalocyanine or azobenzene with alkyl chains, where we expect a similar behavior.

Conclusions. By choosing a QA molecule as a model system, we have revealed the detailed deformation processes of an organic film. We evaluated the contributions of the various molecular interactions and found that such a subtle process should be considered as a collective interaction between molecules and substrates rather than from the viewpoint of an isolated molecule. In particular, the energy per unit area obtained by considering all possible interactions (including weak vdW forces) represents an effective surface energy, which offers an efficient tool to evaluate the stability and evolution of molecular configurations of the film. Complicated interactions in monolayers and their subtle cooperation and competition revealed here should be helpful for the understanding of structures and properties of films in real devices. Importantly, we show that mechanical properties can be evaluated from STM experiments combined with theoretical calculations. The Young's modulus of our QA system is substantially smaller than those of typical organic-molecule based crystals (e.g., naphthalene) due to the long carbon side chains. The possibility of using alkyl side chains to control molecular configurations offers a new avenue to tune the elastic properties of organic films, which is anticipated to be an important guide for future development of functional molecular devices.

\section{ASSOCIATED CONTENT}

\section{S Supporting Information}

Self-assembled patterns of QA16C on $\mathrm{Ag}(110)$ with increasing coverage (S1); molecular packing density of eight observed structures (S2); self-assembled patterns of QA1C and QA4C molecules on $\operatorname{Ag}(110)$ (S3); adsorption configurations of molecular backbone on $\operatorname{Ag}(110)$ (S4); thermally driven structural evolution of QA16C film (S5); definition of the total number of carbon atoms per side chain adsorbed on surface (S6); elastic property calculations and results (S7); parameters of the eight structures of QA16C on $\mathrm{Ag}(110)$ (Table S1); adsorption energies of carbon chains on $\mathrm{Ag}(110)$ (Table S2); adsorption energies of backbones on $\operatorname{Ag}(110)$ (Table S3); total adsorption energies of eight structures (Table S4). This material is available free of charge via the Internet at http://pubs.acs.org.

\section{AUTHOR INFORMATION}

Corresponding Author

*E-mail: (Y.W.) ylwang@iphy.ac.cn; (S.D.) sxdu@iphy.ac.cn.

\section{Author Contributions}

${ }^{\mathrm{II}}$ These authors contribute equally to this work.

\section{Notes}

The authors declare no competing financial interest.

\section{ACKNOWLEDGMENTS}

The authors thank L. Bartels and K.-H. Ernst for fruitful discussions. This works supported by NSFC, MOST, CAS, and SSC in China. M.O. acknowledges support from U.S. National Science Foundation (Career Award). W.A.H. thanks the Royal Society London for support. S.J.P. was supported by the U.S.
Dept. of Energy, Office of Science, Materials Sciences and Engineering Division.

\section{REFERENCES}

(1) Rogers, J. A. Science 2001, 291, 1502-1503.

(2) Forrest, S. R. Nature 2004, 428, 911-918.

(3) Menard, E.; et al. Chem. Rev. 2007, 107, 1117-1160.

(4) Berggren, M.; Nilsson, D.; Robinson, N. D. Nat. Mater. 2007, 6, $3-5$.

(5) Kassner, M. E.; et al. Mech. Mater. 2005, 37, 231-259.

(6) Smith, W. F. Nat. Nanotechnol. 2007, 2, 77-78.

(7) Sun, D. Z.; et al. Phys. Rev. B 2010, 82, 201410.

(8) Bartels, L. Nature Chem. 2010, 2, 87-95.

(9) Barth, J. V.; Costantini, G.; Kern, K. Nature 2005, 437, 671.

(10) Nakahara, H.; Kitahara, K.; Nishi, H.; Fukuda, K. Chem. Lett. 1992, 5, 711-714.

(11) Lincke, G. Dyes Pigm. 2000, 44, 101-122.

(12) Paulus, E. F.; Leusen, F. J. J.; Schmidt, M. U. CrystEngComm 2007, 9, 131-143.

(13) Service, R. F. Science 2005, 310, 1762-1763.

(14) Gao, L.; et al. Phys. Rev. B 2006, 73, 075424.

(15) McGhie, A. R.; Garito, A. F.; Heeger, A. J. J. Cryst. Growth 1974, 22, 295-297.

(16) Perdew, J. P.; et al. Phys. Rev. B 1992, 46, 6671-6687.

(17) Vanderbilt, D. Phys. Rev. B 1990, 41, 7892-7895.

(18) Kresse, G.; Furthmuller, J. Phys. Rev. B 1996, 54, 11169-11186.

(19) Dion, M.; Rydberg, H.; Schroder, E.; Langreth, D. C.; Lundqvist, B. I. Phys. Rev. Lett. 2004, 92, 246401.

(20) Cun, H. Y.; et al. Langmuir 2010, 26, 3402-3406.

(21) De Feyter, S.; Gesquiere, A.; De Schryver, F. C.; Keller, U.; Mullen, K. Chem. Mater. 2002, 14, 989-997.

(22) Ye, K.; et al. J. Phys. Chem. B 2005, 109, 8008-8016.

(23) Lin, F.; Zhong, D. Y.; Chi, L. F.; Ye, K.; Wang, Y.; Fuchs, H. Phys. Rev. B 2006, 73, 235420.

(24) Hu, W.; et al. Adv. Mater. 2007, 19, 2119.

(25) Yang, X.; Wang, J.; Zhang, X.; Wang, Z.; Wang, Y. Langmuir 2007, 23, 1287-1291.

(26) Yang, B.; et al. J. Am. Chem. Soc. 2010, 132, 10440-10444.

(27) Tahara, K.; Okuhata, S.; Adisoejoso, J.; Lei, S.; Fujita, T.; Feyter, S. D.; Tobe, Y. J. Am. Chem. Soc. 2009, 131, 17583-17590.

(28) Simmons, G. W. H. Single crystal elastic constants and calculated aggregate properties: A handbook; The MIT Press: Cambridge, 1971; pp 133-311.

(29) Roberts, R. J.; Rowe, R. C. Powder Tech. 1991, 65, 139.

(30) Pawin, G.; Wong, K. L.; Kwon, K. Y.; Bartels, L. Science 2006, 313, 961-962.

(31) Wang, Y.; Lingenfelder, M.; Classen, T.; Costantini, G.; Kern, K. J. Am. Chem. Soc. 2007, 129, 15742.

(32) Tao, F.; Bernasek, S. L. J. Am. Chem. Soc. 2005, 127, 1275012751.

(33) Wei, Y. H.; Tong, W. J.; Wise, C.; Wei, X. L.; Armbrust, K.; Zimmt, M. J. Am. Chem. Soc. 2006, 128, 13362-13363.

(34) Xiao, W. D.; Feng, X. L.; Ruffieux, P.; Groning, O.; Mullen, K.; Fasel, R. J. Am. Chem. Soc. 2008, 130, 8910.

(35) Palma, C. A.; Bjork, J.; Bonini, M.; Dyer, M. S.; Llanes-Pallas, A.; Bonifazi, D.; Persson, M.; Samori, P. J. Am. Chem. Soc. 2009, 131, 13062-13071. 